\title{
The V-Quick patch versus the standard 12-lead ECG system: time is the essence
}

\author{
F. Lateef • A. Annathurai • T. T. Loh
}

Received: 16 January 2008 / Accepted: 5 February 2008/Published online: 15 March 2008

(C) Springer-Verlag London Ltd 2008

\begin{abstract}
Introduction The V-Quick patch template system is compared with the standard 12-lead electrocardiogram (ECG) acquisition technique in this paper. The objectives of the study were: (a) to study and compare the time taken to produce the printed 12-lead ECG and (b) to look at the level of agreement when the ECGs were compared by two blinded, independent assessors.

Methods One hundred and fifty each of male and female volunteers signed an informed consent form to participate in the clinical study. Nurses were put through a 4-h training session to familiarise themselves with the V-Quick patch system. The timings were measured with a stopwatch with the specific start and end points defined. The final ECG printouts were then compared by two blinded, independent assessors for several set criteria.

Results The V-Quick patch system was proved to be significantly faster than the standard 12-lead system in the acquisition of the ECG in both male and female volunteers. The time taken in male volunteers was also noted to be significantly faster than in female volunteers.

Conclusion The two assessors shared a 100\% agreement level when comparing the ECGs acquired by both techniques in the same individual (intra-assessor agreement) and when each ECG was read by the two assessors separately (inter-assessor agreement).
\end{abstract}

Keywords 12 -lead ECG $\cdot$ Praecordial leads $\cdot$ V-Quick patch

Disclaimer: The views expressed in this paper are those of the author(s), and not those of the editors, editorial board or publisher.

F. Lateef $(\bowtie) \cdot$ A. Annathurai $\cdot$ T. T. Loh

Department of Emergency Medicine, Singapore General Hospital,

Outram Road, Singapore 169608, Singapore

e-mail: fatimah.abd.lateef@sgh.com.sg

\section{Introduction}

For patients with acute coronary syndrome (ACS), the key to increased survival and the preservation of myocardial function is rapid diagnosis followed by appropriate early intervention, which will enable timely management strategies and intervention to be carried out. Delays at any level of management, from the prehospital setting to the Emergency Department (ED) and, subsequently, to the cardiac catheterisation laboratory, must be minimised. The diagnosis of acute myocardial infarction requires the combination of a good and prompt clinical history, a 12-lead electrocardiogram (ECG) and also appropriate cardiac markers (in cases of ST-elevation AMI [STEMI], the results of cardiac markers will not be required for decisions on management and intervention) [1-5].

Prehospital 12-lead ECGs are now being performed and transmitted to EDs to save precious time in diagnosis. Institutions set targets where the door-to-ECG time should not exceed $10 \mathrm{~min}$, the door-to-needle time must be within $30 \mathrm{~min}$ and the door-to-intervention time should be within 90 min of arrival [6-11]. The acquisition of a 12-lead ECG is an important step to assess the cardiac status in routine and emergency situations. In a cardiac emergency, the time and accuracy of information are important elements that have a critical effect on patient outcomes. Accurate ECG measurements are essential for diagnostic purposes, as well as serial comparison to evaluate changes in cardiac status and help in the prediction of future events. In clinical practice, the relevance of the ECG depends upon its reproducibility in an individual. With all of these points in mind, every minute counts [12-15].

The application of single-lead electrodes to the torso for standard 12-lead ECG acquisition takes time. Its accuracy is also operator-dependent. A relatively new praecordial V1- 
V6, V-Quick Patch, has been developed and it provides a one-piece template for the placement of all six praecordial leads, which makes application easy. The person performing the 12-lead ECG need only to reach out for one single item to be applied to the patient's torso, instead of six separate pieces of items, as in the traditional case. The patch has characteristics that enable it to conform to variations in thoracic dimensions while retaining the ability of providing accurate electrode placement. All electrodes are pre-gelled for simple peel-and-stick application. Training in the use of this new patch requires a minimal amount of time, as the template provides cues to the user on placement as it is being performed. The V-Quick patch has the potential to be used anywhere and in any setting where a 12-lead ECG is carried out.

\section{Objectives}

This study will assess:

- The time required to obtain a 12-lead ECG using the VQuick patch system versus the standard single-electrode system

- The agreement between the 12-lead ECGs acquired by both techniques as assessed by two assessors

The null hypothesis would be that there is no difference between the times taken and the level of agreement or quality of the ECGs acquired by the two different techniques.

\section{Methods}

Recruitment and procedure

One hundred and fifty each of healthy male and female volunteers, at least 18 years of age, with various body builds and weights were enrolled. The study did not pose any risks to the volunteers. It was done on a voluntary capacity with informed consent given. Any volunteer who was incidentally found to have significant ECG changes were given an appointment with a cardiologist for the necessary evaluation.

Each volunteer had their ECGs carried out by the two techniques. Preparation consisted of cleaning the skin with alcohol wipes at the site for lead placement. Electrodes were placed in the routine standard positions for the singleelectrode 12-lead ECG. These positions were marked on the praecordium with a skin marker. Following the removal of the standard electrodes, the V-Quick template was attached to the praecordium for the acquisition of the next 12-lead ECG. The volunteers were randomised to see which technique they were to undergo first. This was done using their unique identification number (IN) and if this number ended with an even digit, they would have the VQuick technique ECG performed first. This is, thus, a randomised control trial.

The volunteers may experience some minor dermatological effects from the applications and removal of the electrodes. This may be in the form of erythema or skin irritation. These effects were temporary, with no long-term significant sequelae.

The time was measured with a stopwatch from the instance the lead application commenced for both techniques. For the V-Quick technique, this would be from the moment the staff tears the pack open and for the standard method, it would be from the time the staff picks up the card of the single-electrode adhesive. The stopwatch was stopped once the last electrode had been applied satisfactorily and the ECG printout was complete. The timing was carried out by an independent nursing manager. The two ECGs acquired by the different techniques, on the same volunteer, was performed by the same staff.

The two ECGs were then compared in a blinded fashion by two senior emergency physicians. Both the interassessor (consistency between the two readers of the ECGs) and intra-assessor (same interpretation for both ECGs in the same volunteer) agreement were analysed. The intraassessor agreement reflected the similarity between the two techniques being studied.

\section{Exclusion criteria}

The following cases were excluded:

- Known electrolytes abnormalities or ingestion of medication which had the potential to cause electrolyte imbalance (e.g. diuretics, potassium supplements)

- Congestive heart failure

- History of renal and hepatic impairment

- Acute illness on the day that the ECGs were to be acquired

- Ingestion of medication which could affect the heart rate and rhythm

- The presence of dermatological disease which may affect the skin sites for electrode application

- Implanted pacemaker or automated implanted cardiac defibrillator (AICD)

These patients were excluded because this was the first time we were using the V-Quick device in Singapore and there was no prior literature available on its use in Asian patients and also because of how long it was going to take our nurses to obtain the 12-lead ECG. Despite the null hypothesis of no difference for both techniques, we decided to first perform this trial on a normal, healthy cohort of 
volunteers. In the next phase, we plan to include all patients requiring $\mathrm{ECG}$ presenting to the $\mathrm{ED}$.

\section{The ECG machine}

An Agilent Pagewriter 300PI and Hewlett Packard Pagewriter $\mathrm{XLi}$ was used to obtain all of the ECGs. These machines utilise a computerised analysis system and were the ones used in the ED.

The V-Quick patch template

The V-Quick patch system is packaged with one guide template with the four replaceable limb lead electrodes. The electrodes were pre-gelled for a simple peel-and-stick application. The guide template has the flexibility to accommodate different body sizes and geometries by allowing the movement of each (praecordial) electrode along the guide to attain the best suit for the recommended position (Figs. 1 and 2). The staff involved were put through a 4-h training programme for familiarisation.

\section{Data management and ECG analyses}

SPSS version 10.0 (SPSS Inc., Chicago, IL) was used for the data entry and statistical analyses. Demographic data was managed using descriptive statistics. $p$-values and confidence intervals were analysed using Student's $t$-test. In the collection of data, some of the information gathered included:

- Bra cup size for ladies: this was thought to be a good subjective assessment of breast size and volume

- Shirt size for men: this would give an approximate indication of the shoulder width

- Distance between leads V2-V4 and V4-V6: these measurements may indicate how broad the chest is or

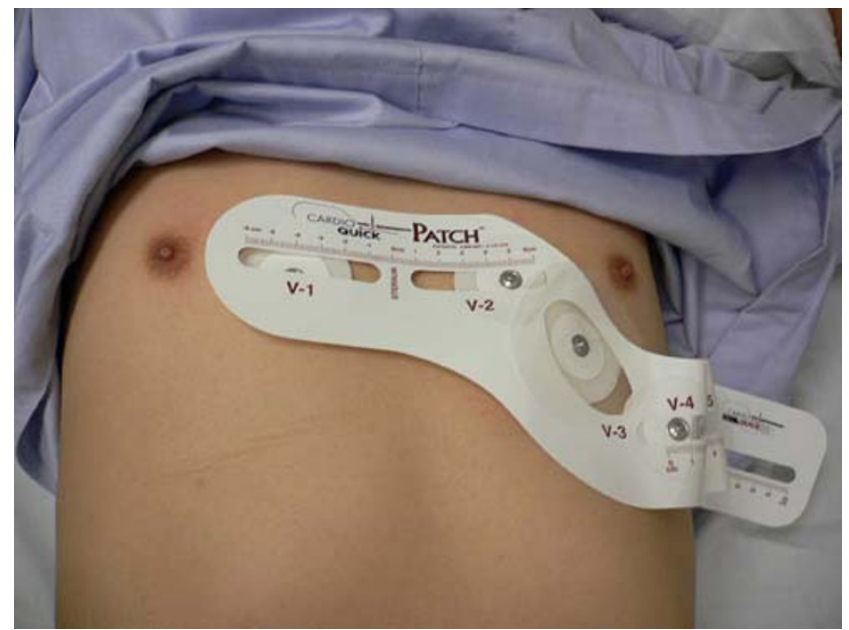

Fig. 1 The V-Quick patch placement system

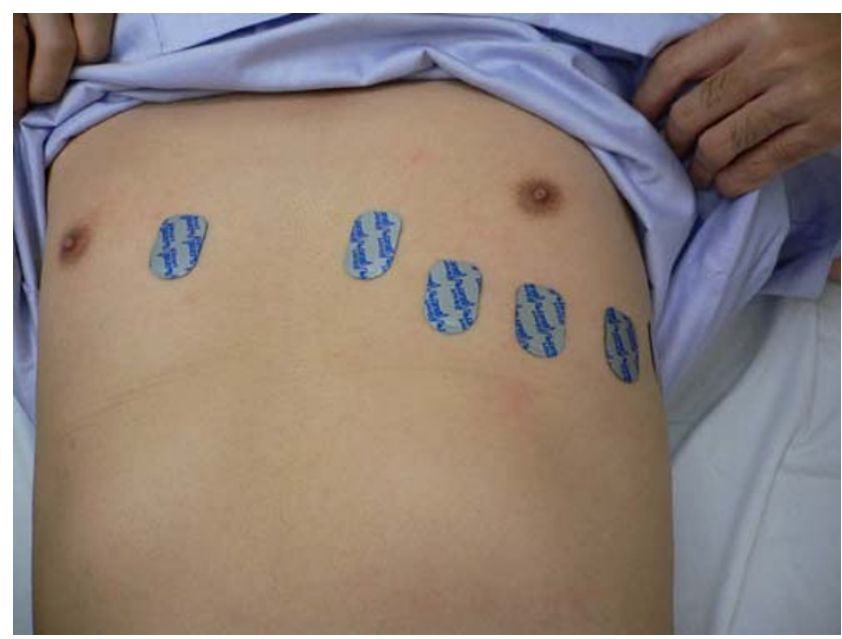

Fig. 2 The standard single-lead electrocardiogram (ECG) system

the expanse of the chest over the area where the ECG electrodes are placed

The ECGs were analysed and compared in a blinded fashion by the two assessors. The following were examined:

- Heart rate and rhythm

- Special patterns, such as bundle branch block and ventricular hypertrophy

- R-wave amplitude and R-wave progression

- S-wave depth

- Presence of a Q-wave and its morphology

The study was approved by the Institutional Review Board and each volunteer was given a written informed consent form to sign for participation in the study. The details were explained verbally to the prospective volunteers also.

\section{Results}

The mean ages for the male and female volunteers were $43 \pm 10.8$ and $52 \pm 12.7$ years, respectively, with the female

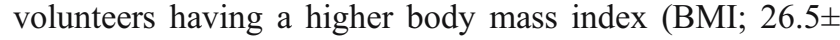
4.2 versus $24.3 \pm 5.8, p=0.0003$ ) (Table 1 ). The race distribution is also shown in Table 1.

When comparing the mean time taken for both techniques, the V-quick method proved to be faster in both the male ( $40.5 \pm 6.7$ versus $58.6 \pm 10.8 \mathrm{~s}, p<0.0001)$ and female volunteer $(50.2 \pm 5.7$ versus $66.0 \pm 12.2 \mathrm{~s}, p<0.0001)$ groups (Table 2). Even when the male and female volunteers were taken together (i.e. all 300 volunteers were compared for the two techniques), the V-Quick method was also shown to be significantly faster $(45.4 \pm 3.8$ versus $61.9 \pm 3.5 \mathrm{~s}$, $p<0.0001$ ) (Table 2).

When the different methods were compared between the genders (Table 3), the V-Quick method was shown to be 
Table 1 Demographic and comparison data between the male and female volunteers

\begin{tabular}{|c|c|c|c|}
\hline & & Male $(n=150)$ & $\begin{array}{l}\text { Female } \\
(n=150)\end{array}$ \\
\hline \multicolumn{2}{|l|}{ Age range } & $19-68$ & $23-72$ \\
\hline \multicolumn{2}{|l|}{ Mean age } & $43 \pm 10.8$ & $52 \pm 12.7$ \\
\hline \multirow[t]{4}{*}{ Race } & Chinese & 108 & 95 \\
\hline & Malay & 20 & 15 \\
\hline & Indian & 18 & 27 \\
\hline & Other & 4 & 13 \\
\hline \multicolumn{2}{|l|}{ BMI range } & $18.9-39.5$ & $17.5-38.8$ \\
\hline \multicolumn{2}{|l|}{ Mean } & $24.3 \pm 5.8$ & $26.5 \pm 4.2$ \\
\hline \multirow[t]{2}{*}{ Standard method } & Mean time (s) & $58.6 \pm 10.8$ & $66.0 \pm 12.2$ \\
\hline & Range & $44.9-68.5$ & $54.0-82.1$ \\
\hline \multirow[t]{2}{*}{ V-Quick method } & Mean time (s) & $40.5 \pm 6.7$ & $50.2 \pm 5.6$ \\
\hline & Range & $35.1-45.9$ & $41.3-53.9$ \\
\hline \multicolumn{2}{|c|}{ V2-V4 distance $(\mathrm{cm})$} & 12.4 & 9.2 \\
\hline \multicolumn{2}{|c|}{ V4-V6 distance $(\mathrm{cm})$} & 10.6 & 9.8 \\
\hline \multicolumn{2}{|c|}{ Range of shirt size } & $15-24$ & \\
\hline \multirow{4}{*}{\multicolumn{2}{|c|}{ Range of bra cup size }} & & A: 20 \\
\hline & & & B: 47 \\
\hline & & & C: 69 \\
\hline & & & D: 14 \\
\hline
\end{tabular}

significantly faster in male volunteers compared to female volunteers. The same results were also noted for the standard method; it was faster to acquire the 12-lead ECG by the standard method in male volunteers.

In the blinded analyses of the ECGs, there was 100\% agreement between the two assessors when comparing ECGs of the same person (in both male and female volunteers) acquired by the two different techniques (intra-assessor agreement) and when each ECG was read by the two assessors separately (inter-assessor agreement). Both assessors agreed in all categories of ECG assessment mentioned in the Section 3.

\section{Discussion}

The ECG continues to be a critical component of the evaluation of patients with emergency cardiac symptoms. This tool is now more than a hundred years old and has

Table 2 Comparison of the methods with $p$-values

\begin{tabular}{cccc}
\hline & $\begin{array}{l}\text { Standard } \\
\text { method }\end{array}$ & $\begin{array}{l}\text { V-Quick } \\
\text { method }\end{array}$ & $\begin{array}{l}p \text {-values } \\
(95 \% \mathrm{CI})\end{array}$ \\
\hline $\begin{array}{c}\text { Mean time for all } \\
\text { 300 volunteers (s) }\end{array}$ & $61.9 \pm 3.5$ & $45.4 \pm 3.8$ & $\begin{array}{c}p<0.0001 \\
(15.922,17.078)\end{array}$ \\
$\begin{array}{c}\text { Mean time for 150 } \\
\text { female volunteers (s) }\end{array}$ & $66.0 \pm 12.2$ & $50.2 \pm 5.7$ & $\begin{array}{c}p<0.0001 \\
(13.636,17.964)\end{array}$ \\
$\begin{array}{c}\text { Mean time for 150 } \\
\text { male volunteers (s) }\end{array}$ & $58.6 \pm 10.8$ & $40.5 \pm 6.7$ & $\begin{array}{c}p<0.0001 \\
(16.058,20.142)\end{array}$ \\
\hline
\end{tabular}

Table 3 Comparison between genders of the two methods

\begin{tabular}{llll}
\hline & Male & Female & $p$-values $(95 \% \mathrm{CI})$ \\
\hline Mean time for & $58.6 \pm 10.8$ & $66.0 \pm 12.2$ & $\begin{array}{c}p<0.0001 \\
(-10.018,-4.782)\end{array}$ \\
standard method (s) & & & $p<0.0001$ \\
Mean time for & $40.5 \pm 6.7$ & $50.2 \pm 5.7$ & $(-11.113,-8.287)$ \\
V-Quick method (s) & & & \\
\hline
\end{tabular}

been a standard in clinical practice for more than half a century. The applications of new signal processing techniques and the expansion of the use of additional leads and innovative techniques have offered more information on cardiac electrical activity [16].

Even though it is one of the most common tests being performed today, there are hardly any published studies on the time taken to acquire it. Often, ECGs are acquired by ECG technicians or nurses who, over the years, have become very versatile and familiar with the importance of accurate lead placement according to the thoracic landmarks. There has also been developments with the aim of making praecordial lead placement easier and faster. One of these is the ECG BELT [17-18].

The V-Quick technique showed a favourable result from this study in terms of timing, as well as agreement with the standard 12-lead ECG in 300 normal volunteers. The technique is simple and the skill relatively easy to acquire when one already knows the concept of the 12-lead ECG. The one-piece template and self-adhesive nature are also plus points. The fact that the template is manufactured to fit the average standard size adult torso will make adjustment rarely necessary. It is also accommodative to both genders. However, having said that, the onus will still lie with the operator carrying out the ECG to strive for the most accurate placement.

Besides accuracy, timing is the other important factor, as we all try to meet the targets of door-to-ECG time at our institutions. It is important to highlight that there are multiple factors that affect this timing: how busy the department is at any particular point in time, the first contact time or triage time, manpower and staffing issues, as well as the availability of ECG machines throughout the ED, among other factors. It is, thus, crucial for each institution to look at the wider picture when trying to address timing issues to meet key performance indicators (KPIs).

In female volunteers, the longer time required can be explained by the presence of breast tissue, which requires more attention to be paid to the standard landmarks and whether the leads should be placed over or under the breast tissue. Comparatively, with one template to deal with, it is still faster to apply the V-Quick system rather than to apply the leads singly over the praecordium, as shown in this study. 
In this study, the V-Quick system gave a stable baseline on ECG recordings, unlike some techniques such as the ECG BELT, where this was a common problem [17].

Praecordial electrode position is important, as the variability in placement may result in ECG changes. Here, the nurses performing the ECGs on every individual marked the points of application with a skin marker to reduce the variability in placement between the two techniques. The limb leads were also placed on the usual suggested limb positions in this study. There has been increasing practice to place the limb leads on the torso itself, as this has been thought to reduce the application time. However, it has been shown to have significant amplitude and waveform changes when compared to the ECG acquired with the limb leads placed in the usual recommended limb positions [19,20].

This trial was a first of its kind conducted in Singapore using the V-Quick patch system. It was tested on stable volunteers in the ED. Looking at the results and also from past experience of the utilisation of the system, it appears to be very useful for consideration for use in the prehospital environment, where conditions are more unpredictable and stressful. As it is relatively easy to apply, using it whilst in a moving ambulance will also be less of a challenge when compared to the application of the small, single adhesives that the paramedics would have to apply at six different sites over the praecordium. Many prehospital patients are also cold, clammy and sweaty and, very often, the adhesives from these small electrodes do not work well. This is where the one, single, larger template of the VQuick system would be a plus point.

We will, in future, consider a trial using the V-Quick system in all patients who present to the ED and also the prehospital group of patients requiring a 12-lead ECG.

\section{Limitations and observations}

The V-Quick patch was manufactured by a US-based company and it is likely the standardisation of the template was for the average American torso size. In this study, the template system was applied to Asian volunteers. In the application, adjustments had to be made to the template, thus, creating more "curves" along the template (Fig. 1). This may have been due to the slightly smaller chest expanse of the average Asian compared to their Caucasian counterpart. In the data collection, the distances between V2-V4 and V4-V6 were noted, as these were thought to be useful as a simple measure of chest or thoracic expanse. The shirt size may too be a reflection of this, but may be less constant or objective, as it would be very much affected by personal choice. The same goes for female volunteers, where the bra cup size may reflect breast tissue size and volume. If such similar studies were to be conducted in the Caucasian population, it would be interesting to see the results and compare them to ours.

In this trial, the nurses performing the ECGs were not blinded, as this would have been impossible to achieve. The nursing manager carrying out the timing was also not blinded. It would have been optimal to do so, except for the fact that, logistically, it was rather challenging and would have incurred the extra cost of videotaping.

\section{Conclusion}

In this study of 300 male and female volunteers, the V-Quick patch system was proved to be significantly faster than the single-electrode standard 12-lead system for the acquisition of electrocardiograms (ECGs). The time taken was also faster in male compared to female volunteers for the reasons discussed. Two independent assessors had 100\% agreement when analysing these ECGs in a blinded fashion.

Acknowledgement The investigators would like to thank CardioQuickSys, LLC, a subsidiary of the VQ Company, USA, for providing the equipment and disposable supplies required to perform this clinical evaluation. There were no monetary transactions between the company and any of the investigators or the institution.

\section{References}

1. Gomes S, Pereira D, Oliveira R et al (2005) New diagnostic criteria for acute myocardial infarction and in-hospital mortality. Rev Port Cardiol 24(2):231-237

2. Pell JP, Simpson E, Rodger JC et al (2003) Impact of changing diagnostic criteria on incidence, management, and outcome of acute myocardial infarction: retrospective cohort study. BMJ 326:134-235

3. World Health Organization Expert Committee (1959) Hypertension and coronary heart disease: classification and criteria for epidemiological studies. World Health Organization, Geneva, Technical Report Series, no. 168

4. Hamm CW, Ravkilde J, Gerhardt W et al (1992) The prognostic value of serum troponin $\mathrm{T}$ in unstable angina. $\mathrm{N}$ Engl $\mathrm{J}$ Med 327:146-150

5. [No authors listed] (2002) Myocardial infarction redefined-a consensus document of The Joint European Society of Cardiology/ American College of Cardiology Committee for the redefinition of myocardial infarction. Eur Heart J 21:1502-1513

6. Dorsch MF, Lawrence RA, Sapsford RJ et al (2001) A simple benchmark for evaluating quality of care of patients following acute myocardial infarction. Heart 86:150-154

7. Thatcher JL, Gilseth TA, Adlis S (2003) Improved efficiency in acute myocardial infarction care through commitment to emergency department-initiated primary PCI. J Invasive Cardiol 15: 693-698

8. Diercks DB, Kirk JD, Lindsell CJ et al (2002) Compliance with guidelines for door-to-ECG time in patients with chest pain suspicious for myocardial ischemia. Acad Emerg Med 9(5): 371-372 
9. Parish K, Brown J, Ross MA et al (2006) Triaged-based electrocardiogram process reliably decreases time to ST-elevation myocardial infarction diagnosis. Acad Emerg Med 13(Suppl I):33-34

10. Bradley EH, Herrin J, Wang YF et al (2006) Door-to-drug and door-to-balloon times: where can we improve? Time to reperfusion therapy in patients with ST-segment elevation myocardial infarction (STEMI). Am Heart J 151:1281-1287

11. Bradley EH, Roumanis SA, Radford MJ et al (2005) Achieving door-to-balloon times that meet quality guidelines: how do successful hospitals do it? J Am Coll Cardiol 46:1236-1241

12. Cannon CP, Gibson CM, Lambrew CT et al (2000) Relationship of symptom-onset-to-balloon time and door-to-balloon time with mortality in patients undergoing angioplasty for acute myocardial infarction. JAMA 283:2941-2947

13. Berger PB, Ellis SG, Holmes DR Jr et al (1999) Relationship between delay in performing direct coronary angioplasty and early clinical outcome in patients with acute myocardial infarction: results from the global use of strategies to open occluded arteries in Acute Coronary Syndromes (GUSTO-IIb) trial. Circulation 100:14-20
14. Angeja BG, Gibson CM, Chin R et al (2002) Predictors of doorto-balloon delay in primary angioplasty. Am J Cardiol 89:11561161

15. Pope C, Mays N (1995) Reaching the parts other methods cannot reach: an introduction to qualitative methods in health and health services research. BMJ 311:42-45

16. Garvey JL (2006) ECG techniques and technologies. Emerg Med Clin North Am 24(1):209-225

17. Bell SJ, Clifton J, Pease J et al (2001) The evaluation of a precordial ECG BELT: technologist satisfaction and accuracy of recording. J Electrocardiol 34(2):155-159

18. Mills H, Stein HI, Mandel WJ (1979) The precordial ECG BELT for obtaining rapid reproducible precordial leads. J Electrocardiol 12(4):407-410

19. Sheffield LT, Roitman DI, Kansal S (1978) A quantitative technical quality assay method for electrocardiograms. J Electrocardiol 11(3):277-283

20. Jowett NI, Turner AM, Cole A et al (2005) Modified electrode placement must be recorded when performing 12-lead electrocardiograms. Postgrad Med J 81(952):122-125 